\title{
THE GENUS SPECTRUM OF A HYPERBOLIC 3-MANIFOLD
}

\author{
D.B. McReynolds And A.W. ReID
}

\begin{abstract}
In this paper, we study the spectrum of totally geodesic surfaces of a finite volume hyperbolic 3-manifold. We show that for arithmetic hyperbolic 3-manifolds that contain a totally geodesic surface, this spectrum determines the commensurability class. In addition, we show that any finite volume hyperbolic 3-manifold has many pairs of non-isometric finite covers with identical spectra. Forgetting multiplicities, we can also construct pairs where the volume ratio is unbounded.
\end{abstract}

\section{Introduction}

Let $M$ be a closed orientable Riemannian manifold $M$. An invariant of $M$ that has traditionally been of great importance in understanding the geometry of $M$ is the geodesic length spectrum (that is the set of lengths of closed geodesics counted with multiplicities). For example, in the case when $M$ admits a metric with all sectional curvatures negative, there is a strong relationship between this spectrum and the eigenvalue spectrum of the Laplace-Beltrami operator $[2,3]$; the latter is well known to determine the volume and dimension of $M$. The aim of this paper is to begin the development of more general geometric spectra for Riemannian manifolds.

In more detail, the geodesic length spectrum encodes isometric immersions of $S^{1}$ into $M$, and in this paper we will study the two-dimensional case; that is totally geodesic immersions of orientable, finite-type surfaces into Riemannian manifolds. One can certainly consider higher dimensional submanifolds but for simplicity we have elected to only concern ourselves with surfaces. Motivated by the case of the length spectrum two natural (complementary) questions are:

Question 1. How much of the geometry of $M$ is determined by the totally geodesic surfaces immersed in $M$ ?

Question 2. Do there exist non-isometric Riemannian manifolds with the same "spectra" of totally geodesic surfaces?

To state our results we describe the set up more carefully. We will consider only the case when the manifold $M$ is a complete orientable hyperbolic 3-manifold of finite volume. Let $\Sigma_{g, n}$ denote the closed orientable surface of genus $g$ with $n$ punctures and let $M$ be as indicated above. For each finite-type hyperbolic surface $X$ in the moduli space of such a surface $\Sigma_{g, n}$, set $\ell_{X}(M)$ to be the number of the free homotopy classes of totally geodesic surfaces in $M$ with a representative isometric to $X$. That $\ell_{X}(M)$ is finite follows from [19] Corollary 8.8.6. We define the geometric genus spectrum to be the set of pairs

$$
\mathcal{G} S(M)=\left\{\left(X, \ell_{X}(M)\right): \ell_{X}(M) \neq 0\right\} .
$$

Received by the editors August 21, 2013. 
We say that $M_{1}$ and $M_{2}$ are geometrically isogenus if $\mathcal{G} S\left(M_{1}\right)=\mathcal{G} S\left(M_{2}\right)$. Forgetting the multiplicities $\ell_{X}(M)$ in $\mathcal{G S}(M)$ leads to the geometric genus set given by

$$
\operatorname{GS}(M)=\left\{X: \ell_{X}(M) \neq 0\right\} .
$$

We say that $M_{1}$ and $M_{2}$ are geometrically genus equivalent if $\operatorname{GS}\left(M_{1}\right)=\operatorname{GS}\left(M_{2}\right)$.

Our first result addresses the degree to which the geometric genus set or spectrum governs the geometry of $M$.

Theorem 1.1. Let $M_{1}=\mathbf{H}^{3} / \Gamma_{1}$ and $M_{2}=\mathbf{H}^{3} / \Gamma_{2}$ be arithmetic hyperbolic 3-manifolds. If $M_{1}$ and $M_{2}$ are geometrically genus equivalent then either $G S\left(M_{1}\right)=$ $\emptyset$, or $M_{1}$ and $M_{2}$ are commensurable.

The first possibility does occur since, unlike the case of the length spectrum, the set $\mathcal{G} S(M)$ can be empty; indeed, most finite volume hyperbolic 3-manifolds do not contain an immersed totally geodesic surface (see [11] Chapter 5.3). However, and perhaps most interestingly from the point of view of this spectrum, if an arithmetic hyperbolic 3-manifold contains one totally geodesic surface, it contains infinitely many such surfaces (up to commensurability). We do not know if this holds in general (see Section 8 for a further discussion of this). Thus arithmetic manifolds provide a good class to analyze in regard to geometric spectra. It is also worth remarking that even in the case of the length spectrum, there are no comparable results to Theorem 1.1 without an arithmetic assumption — see [17, 1, 16].

Totally geodesic submanifolds and their relation to the geometry of the ambient manifold were studied in Prasad-Rapinchuk [16] where the submanifolds were maximal flats. These submanifolds reduce to geodesics when the dimension is one. For a wide class of locally symmetric manifolds, they were able to determine that these submanifolds determine the commensurability class of the ambient manifold. Those results and Theorem 1.1 can be stated in terms of algebraic groups. Specifically, associated to an arithmetic hyperbolic 3 -manifold $M$ is a $K$-form of $\operatorname{SL}(2, \mathbf{C})$ that determines the commensurability class of $M$. The totally geodesic submanifolds correspond to $F$-forms of SL(2, C) over a totally real subfield $F$ of $K$ with $K / F$ quadratic. Theorem 1.1 shows that one can recover the $K$-form from the $F$-forms provided there are some $F$-forms. Alternatively, replacing the $K$-form with the associated $K$-quaternion algebra $A / K$, Theorem 1.1 shows that one can recover $A$ from the $F$-quaternion subalgebras of $A$. In contrast, when $K / F$ is not quadratic, one cannot always recover the algebra $A$. In [13], examples of number fields $K_{1}, K_{2}$, containing a common field $F$ and quaternion algebras $A_{1}, A_{2}$ over $K_{1}, K_{2}$ were constructed with the same $F$-quaternion subalgebras.

As noted above, arithmetic hyperbolic 3-manifolds are exceptional in that they typically do not have totally geodesic surfaces. In higher dimensions, arithmetic hyperbolic $n$-manifolds always have totally geodesic submanifolds beyond just geodesics. Recently, Theorem 1.1 has been extended by Meyer [14] to certain higher dimensional arithmetic hyperbolic $n$-manifolds for $n \geq 4$. More specifically, Meyer[14] extends Theorem 1.1 to arithmetic hyperbolic $n$-manifolds arising from special orthogonal groups of quadratic forms. These manifolds account for all commensurability classes in even dimensions. However, in odd dimensions there are additional commensurability classes. 
More generally, locally symmetric manifolds typically have a rich collection of totally geodesic submanifolds and we expect that these submanifolds encode a substantial amount of the geometry of the ambient manifold. In particular, Theorem 1.1 should have an analog for a large class of locally symmetric manifolds.

Similarities with results for the length (and eigenvalue) spectrum are also evident in our two other main results (cf. $[18,9,16])$.

Theorem 1.2. Let $M=\mathbf{H}^{3} / \Gamma$ be an arithmetic hyperbolic 3-manifold. Then there exist infinitely many pairs of finite covers $\left(M_{j}, N_{j}\right)$ of $M$ such that $M_{j}, N_{j}$ are geometrically isogenus and non-isometric.

Theorem 1.3. Let $M=\mathbf{H}^{3} / \Gamma$ be an arithmetic hyperbolic 3-manifold. Then there exist infinitely many pairs of finite covers $\left(M_{j}, N_{j}\right)$ of $M$ such that $M_{j}, N_{j}$ are geometrically genus equivalent and the sequence $\left\{\operatorname{vol}\left(M_{j}\right) / \operatorname{vol}\left(N_{j}\right)\right\}_{j}$ is unbounded.

Though the method of proof for Theorems 1.2 and 1.3 is in the same spirit as Sunada's method for producing iso-length and isospectral manifolds [18], unlike Sunada's method it is not purely algebraic (see the discussion in Section 4.2 for more on this comparison).

Theorems 1.2 and 1.3 also hold without an arithmetic assumption. However, we have elected only to completely treat the case of arithmetic hyperbolic 3-manifolds since, as mentioned above, it is only in this case that we know that the geometric genus spectrum being non-trivial implies that there are infinitely many totally geodesic surfaces. In addition, the proofs in the arithmetic cases are somewhat simpler. In Section 7, we sketch the modifications needed in the proofs for non-arithmetic manifolds.

\section{Preliminaries}

In this section, we collect some preliminary material and notation that we will use throughout. For convenience, we will often blur the distinction between Kleinian groups as subgroups of $\operatorname{SL}(2, \mathbf{C})$ and $\operatorname{PSL}(2, \mathbf{C})$.

Notation: For a number field $K, R_{K}$ will denote the ring of $K$-integers. If $L / K$ is an extension of number fields, $P$ a prime ideal of $R_{K}$, and $\mathfrak{p}$ an ideal of $R_{L}$ such that $\mathfrak{p} \mid P$, we then denote by $\omega_{P}$ (resp. $\nu_{\mathfrak{p}}$ ) the places associated to these primes and write $\nu_{\mathfrak{p}} \mid \omega_{P}$. For a quadratic extension $K^{\prime} / K$ of number fields and a $K$-prime $\mathfrak{p}$, by the splitting type of $\mathfrak{p}$ in $K^{\prime}$, we mean whether $\mathfrak{p}$ is ramified, inert, or split in $K^{\prime} / K$. If $B$ is a quaternion algebra over $K$, we denote the set of finite places of $K$ at which $B$ is ramified by $\operatorname{Ram}_{f} B$.

2.1. For a prime $p \in \mathbf{N}, \mathbf{F}_{q}$ will denote the unique field of order $q=p^{j}$ for each $j \geq 1$ and $\mathbf{F}_{q}(\sqrt{b})$ the unique quadratic extension of $\mathbf{F}_{q}$. In a slight abuse of notation, the undecorated $b$ above represents some element of $\mathbf{F}_{q}$ that is not a square in $\mathbf{F}_{q}$, in essence suppressing its dependence on the $p$ and $j$. As no specific property of $b$ will be used, this abuse is mild. In what follows, to avoid some complications, we will assume $p \neq 2$. 
Associated to each field $\mathbf{F}_{q}$ is the finite group $\operatorname{PSL}\left(2, \mathbf{F}_{q}\right)$ which is well known to have order $\frac{q\left(q^{2}-1\right)}{2}$. The standard Borel subgroup of $\operatorname{PSL}\left(2, \mathbf{F}_{q}\right)$ is given by

$$
\mathbf{B}\left(\mathbf{F}_{q}\right)=\left\{\left(\begin{array}{cc}
\alpha & \beta \\
0 & \alpha^{-1}
\end{array}\right): \alpha \in\left(\mathbf{F}_{q}\right)^{\times} /\{ \pm 1\}, \beta \in \mathbf{F}_{q}\right\}
$$

and has order $\frac{q(q-1)}{2}$.

In our proofs of Theorems 1.2 and 1.3, we will need elements of a prescribed order. This is accomplished with the following well-known lemma.

Lemma 2.1. For each divisor $m$ of $(q \pm 1)$, there exists $\gamma_{m}$ in $\operatorname{SL}\left(2, \mathbf{F}_{q}\right)$ with order $m$.

Proof. For divisors of $q-1$, simply note that we have the inclusion of $\left(\mathbf{F}_{q}\right)^{\times}$into $\mathrm{SL}\left(2, \mathbf{F}_{q}\right)$ given by

$$
\alpha \mapsto\left(\begin{array}{cc}
\alpha & 0 \\
0 & \alpha^{-1}
\end{array}\right)
$$

The existence of $\gamma_{m}$ now follows from the fact that $\left(\mathbf{F}_{q}\right)^{\times}$is cyclic.

For divisors of $q+1$, the ensuing argument produces the desired elements. As right multiplication of $\left(\mathbf{F}_{q}(\sqrt{b})\right)^{\times}$on $\mathbf{F}_{q}(\sqrt{b})$ is $\mathbf{F}_{q}$-linear, there exists an injective homomorphism $\left(\mathbf{F}_{q}(\sqrt{b})\right)^{\times}$into $\operatorname{GL}\left(2, \mathbf{F}_{q}\right)$ given by selecting an $\mathbf{F}_{q}$-basis for $\mathbf{F}_{q}(\sqrt{b})$. For any element $\gamma$ in $\left(\mathbf{F}_{q}(\sqrt{b})\right)^{\times}, \operatorname{det}\left(\gamma^{q-1}\right)=1$ since $\operatorname{det} \gamma \in \mathbf{F}_{q}^{\times}$and determinants are multiplicative. Appealing again to the fact that $\left(\mathbf{F}_{q}(\sqrt{b})\right)^{\times}$is cyclic, we may take $\gamma$ of order $q^{2}-1$ and thus produce an element $\gamma^{s(q-1)}$ in $\operatorname{SL}\left(2, \mathbf{F}_{q}\right)$ of order $m$ where $m s=q+1$.

2.2. We will require some control over the behavior of primes in extensions. The first result of this type we need is on the splitting of primes in quadratic extensions.

Proposition 2.2. Let $K_{1}$ and $K_{2}$ be quadratic extensions of a number field $K$. If all but a finite number of $K$-primes have the same splitting type in $K_{1}$ and $K_{2}$, then $K_{1}=K_{2}$.

Proof. For $i=1,2$, let $S_{i}$ be the set of primes of $K$ that split in $K_{i}$. Since $S_{1}=S_{2}$ apart from a finite number of primes (in particular density 0), we deduce from [6] Chap. IV Corollary 5.5 that $K_{1}=K_{2}$ as required.

Now let $k / L$ be a proper extension of number fields, $\omega$ a finite place of $L$ and $\nu \mid \omega$ a place of $k$. For brevity we simply denote the extension of residue class fields by $\mathbf{F}_{q_{\nu}} / \mathbf{F}_{q_{\omega}}$.

Lemma 2.3. There exists a positive density set of finite places $\nu$ of $k$ such that $\mathbf{F}_{q_{\nu}} / \mathbf{F}_{q_{\omega}}$ is an extension of degree at least 2. Moreover, we can arrange for both $\left[\mathbf{F}_{q_{\nu}}: \mathbf{F}_{q_{\omega}}\right]$ and $\left[\mathbf{F}_{q_{\omega}}: \mathbf{F}_{p_{\nu}}\right]$ to be fixed over this collection of places.

Proof. Let $k_{\text {gal }}$ denote the Galois closure of $k$. As $k \neq L$, there exists a Galois automorphism $\theta$ in $\operatorname{Gal}\left(k_{\text {gal }} / L\right)$ such that $\theta$ is trivial on $L$ and non-trivial on $k$. According to the Chebotarev Density Theorem, there exists a positive density set of places $\nu_{\text {gal }}$ of $k_{\text {gal }}$ such that the induced Galois automorphism $\bar{\theta}$ on the Galois extension $\mathbf{F}_{q_{\nu_{\text {gal }}}}$ of $\mathbf{F}_{q_{\omega}}$ has order $|\theta|$. In particular, $\bar{\theta}$ is trivial on $\mathbf{F}_{q_{\omega}}$ but not $\mathbf{F}_{q_{\nu}}$. Thus $\mathbf{F}_{q_{\nu}} / \mathbf{F}_{q_{\omega}}$ is a proper extension for all the residue fields $\mathbf{F}_{q_{\nu}}, \mathbf{F}_{q_{\omega}}$ associated to the places $\nu$ and $\omega$ where $\nu_{\text {gal }} \mid \nu$ and $\nu_{\text {gal }} \mid \omega$. For the final claim, let $\mathbf{F}_{p_{\nu}}$ denote the prime field 
for $\mathbf{F}_{q_{\nu}}$ (and hence for $\mathbf{F}_{q_{\omega}}$ too). As the possibilities for the degrees $\left[\mathbf{F}_{q_{\nu}}: \mathbf{F}_{q_{\omega}}\right]$ and $\left[\mathbf{F}_{q_{\omega}}: \mathbf{F}_{p_{\nu}}\right]$ range over finite sets as we vary $\nu$, the result follows immediately from the Pigeon Hole Principle and the fact that a finite union of density zero sets is density zero. Specifically, at least one possibility for $\left[\mathbf{F}_{q_{\nu}}: \mathbf{F}_{q_{\omega}}\right]$ and $\left[\mathbf{F}_{q_{\omega}}: \mathbf{F}_{p_{\nu}}\right]$ with $\mathbf{F}_{q_{\nu}} / \mathbf{F}_{q_{\omega}}$ proper occurs with positive density.

2.3. We recall the basic framework of non-elementary Fuchsian subgroups of Kleinian groups. We will not restrict to the arithmetic setting at present, as some of this material will be required for the general discussion in Section 7 .

Let $M=\mathbf{H}^{3} / \Gamma$ be a finite volume hyperbolic 3 -manifold and $S$ a properly immersed orientable totally geodesic surface in $M$. Then corresponding to $S$ is a nonelementary Fuchsian subgroup of $F<\Gamma$ and a round circle $\mathcal{C} \subset \widehat{\mathbf{C}}=\mathbf{C} \cup \infty$ such that $F<\operatorname{Stab}(\mathcal{C}, \Gamma)$ where

$$
\operatorname{Stab}(\mathcal{C}, \Gamma)=\{\gamma \in \Gamma: \gamma \mathcal{C}=\mathcal{C} \text { and } \gamma \text { preserves components of } \widehat{\mathbf{C}} \backslash \mathcal{C}\}
$$

We refer to the groups $\operatorname{Stab}(\mathcal{C}, \Gamma)$ as maximal Fuchsian subgroups of $\Gamma$. Note that the geometric genus spectrum of $M$ is determined by the maximal Fuchsian subgroups of $\Gamma$.

If $F$ is a non-elementary Fuchsian subgroup of a finite co-volume Kleinian group $\Gamma$, then the invariant trace-field $L=k F$ is a real number field and is a subfield of the invariant trace-field $k=k \Gamma$. Furthermore, the invariant algebra $A F / L$ is a $L$ subalgebra of $A \Gamma$ and $A F \otimes_{L} k \cong A \Gamma$ as $k$-quaternion algebras.

Suppose that $A \Gamma$ is ramified at a finite place $\nu$ and $\omega$ is a place of $k F$ such that $\nu \mid \omega$. Then $A F$ is ramified at $\omega$ since

$$
A \Gamma \otimes_{k} k_{\nu} \cong\left(A F \otimes_{L} k\right) \otimes_{k} k_{\nu}
$$

is isomorphic to $\left(A F \otimes_{L} L_{\omega}\right) \otimes_{L_{\omega}} k_{\nu}$ as $k_{\nu}$-quaternion algebras, and so $A F \otimes_{L} L_{\omega}$ must be a division algebra. However, $A F$ can be ramified at other places (see for example the arithmetic case below). On the other hand, if $A F$ is unramified at $\omega$ then it is clear that $A \Gamma$ is unramified at any $k$-place $\nu$ that divides $\omega$.

Let $\nu$ be a place of $k$ at which $A \Gamma$ is unramified. Assume that $\Gamma \subset A \Gamma^{1}$ (the group of elements of norm 1 in $A \Gamma$ ) and assume additionally that under the induced injection of $\Gamma$ into $\mathrm{M}\left(2, k_{\nu}\right)$, the image is conjugate into $\operatorname{SL}\left(2, R_{\nu}\right)$ where $R_{\nu}$ is the ring of $\nu$-adic integers in $k_{\nu}$. Thus, we will assume that $\Gamma$ injects into $\operatorname{SL}\left(2, R_{\nu}\right)$. Note that this is true for all but a finite number of places $\nu$.

Reducing modulo the maximal ideal $\pi R_{\nu}$ of $R_{\nu}$ and taking the central quotient, gives a homomorphism $\phi_{\nu}: \Gamma \rightarrow \operatorname{PSL}\left(2, \mathbf{F}_{q_{\nu}}\right)$. For all but a finite number of places, the Strong Approximation Theorem (see [15] or [20]) ensures that $\phi_{\nu}$ is surjective. For a place $\omega$ of $L$ with $\nu \mid \omega$, the image of $F$ under $\phi_{\nu}$ depends on whether the $A F$ is ramified at the place $\omega$ or not. More specifically, we will prove the following.

Lemma 2.4. In the notation established above, the image of $F$ under $\phi_{\nu}$ is conjugate (over an algebraic closure of $\mathbf{F}_{q_{\nu}}$ ) to either

(a) a subgroup of $\operatorname{PSL}\left(2, \mathbf{F}_{q_{\omega}}\right)$, when $A F$ is not ramified at $\omega$, or

(b) a subgroup of $\mathbf{B}\left(\mathbf{F}_{q_{\omega}}(\sqrt{b})\right)$ when $A F$ is ramified at $\omega$.

Proof. Suppose first that $A F$ is unramified at $\omega$. In this case, $A F \otimes_{L} L_{\omega}$ is isomorphic to $M\left(2, L_{\omega}\right)$, and it is easy to see from this that the image of $F$ under $\phi_{\nu}$ is isomorphic 
to a subgroup of $\operatorname{PSL}\left(2, \mathbf{F}_{q_{\omega}}\right)$ - indeed it will be conjugate over some finite extension of $\mathbf{F}_{q_{\omega}}$ to a subgroup of $\operatorname{PSL}\left(2, \mathbf{F}_{q_{\omega}}\right)$.

The case when $A F$ is ramified at $\omega$ requires some additional discussion. It will be helpful to recall a certain representation of the division algebra $A F \otimes_{L} L_{\omega}$ (see [11] Exercise 2.6(1)). Let $L_{\mathrm{nr}}$ denote the unique unramified quadratic extension of $L_{\omega}$, and ' denote the non-trivial Galois automorphism of $L_{\mathrm{nr}} / L$. Then the unique division algebra over $L_{\omega}$ can be represented as:

$$
B_{\omega}=\left\{\left(\begin{array}{cc}
a & b \\
\pi_{\omega} b^{\prime} & a^{\prime}
\end{array}\right): a, b \in L\right\} .
$$

Hence, $F$ is conjugate (over an algebraic closure of $L_{\omega}$ and hence $k_{\nu}$ ) to a subgroup of the elements of norm 1 in $B_{\omega}$. Indeed, by assumption (see the discussion preceding the lemma), since $F$ is a subgroup of the unique maximal order of $A F \otimes_{L} L_{\omega}$ (see [11] Chapter 6.4), it follows that $F$ is actually conjugate to a subgroup of the norm 1 elements of $\mathcal{O}_{\omega}$, the unique maximal order in $B_{\omega}$. This discussion, together with [11] Exercise $6.4(1)$, shows that $F$ is conjugate to a subgroup of

$$
\left\{\left(\begin{array}{cc}
a & b \\
\pi_{\omega} b^{\prime} & a^{\prime}
\end{array}\right): a, b \in R\right\},
$$

where $R$ is the valuation ring of $L_{\mathrm{nr}}$.

Now observe that the image of $F$ under the homomorphism $\phi_{\nu}$ is, up to conjugacy, described by its image under the natural reduction quotient of $\mathcal{O}_{\omega}$ by its unique maximal ideal (which is informally, the reduction modulo $\pi_{\omega}$ ). This can be seen to have image in a group of upper triangular matrices with entries in the unique quadratic extension of $\mathbf{F}_{q_{\omega}}$. This completes the proof.

\section{Commensurability class rigidity: Theorem 1.1}

Here we will prove Theorem 1.1. This should be compared with the results found in $[17,1,10,16]$ for the length and eigenvalue spectra.

3.1. We begin by specializing the discussion of Section 2.3 to the case of arithmetic Kleinian groups which contain Fuchsian subgroups. For a fuller account see [11] Chapters 5.3 and 9.5 .

Let $\Gamma$ be an arithmetic Kleinian group which contains a non-elementary Fuchsian subgroup $F$. It is known in this case that if $\mathcal{C}$ is the invariant circle of $F$, then $\operatorname{Stab}(\mathcal{C}, \Gamma)$ is a Fuchsian group of finite co-area. Furthermore, the invariant trace-field $L$ of $F$ is a totally real field, $[k \Gamma: L]=2$, and if $B$ is the invariant quaternion algebra of $F$ then (as above), $B \otimes_{L} k \Gamma=A \Gamma$. However, more can be said in this case about precisely which algebras $B$ can arise. We record the following (see [11] Theorem 9.5.5) which will be useful in the sequel.

Theorem 3.1. Let $\Gamma$ be an arithmetic Kleinian group with invariant trace-field $k$ and invariant quaternion algebra $A / k$. Suppose $[k: L]=2$ where $L=k \cap \mathbf{R}$ and $B$ is a quaternion algebra over $L$ ramified at all real places of $L$ except the identity. Then $A \cong$ $B \otimes_{L} k$ if and only if $\operatorname{Ram}_{f} A$ consists of $2 r$ places (possibly zero) $\left\{\nu_{\mathfrak{p}_{1}}, \nu_{\mathfrak{p}_{1}^{\prime}}, \ldots \nu_{\mathfrak{p}_{r}}, \nu_{\mathfrak{p}_{r}^{\prime}}\right\}$ where the $k$-primes $\mathfrak{p}_{i}$ and $\mathfrak{p}_{i}^{\prime}$ satisfy $\mathfrak{p}_{i} \cap R_{L}=\mathfrak{p}_{i}^{\prime} \cap R_{L}=P_{i},\left\{\omega_{P_{1}}, \ldots, \omega_{P_{r}}\right\} \subset \operatorname{Ram}_{f} B$ with $\operatorname{Ram}_{f} B \backslash\left\{\omega_{P_{1}}, \ldots, \omega_{P_{r}}\right\}$ consisting of primes in $R_{L}$ which are inert or ramified in $k / L$. 
3.2. Proof of Theorem 1.1. Recall in the statement of Theorem 1.1, we are assuming that we have a pair of arithmetic hyperbolic 3-manifolds $M_{1}=\mathbf{H}^{3} / \Gamma_{1}$ and $M_{2}=\mathbf{H}^{3} / \Gamma_{2}$ that are geometrically genus equivalent. Our goal is to show that if $\operatorname{GS}\left(M_{1}\right) \neq \emptyset$, then $M_{1}$ and $M_{2}$ are commensurable. For the proof, set $k_{1}, k_{2}$ and $A_{1} / k_{1}, A_{2} / k_{2}$ to be the invariant trace-fields and quaternion algebras of $\Gamma_{1}, \Gamma_{2}$, respectively. With this notation, to show that $M_{1}$ and $M_{2}$ are commensurable it suffices to show that $k_{1} \cong k_{2}$ and $A_{1} \cong A_{2}$ as algebras over $\mathbf{Q}$ (see [11] Theorem 8.4.1).

Since $\Gamma_{1}$ contains a non-elementary Fuchsian subgroup, $k_{1}$ contains a totally real field $L$ with $\left[k_{1}: L\right]=2$. Furthermore, since $M_{1}$ and $M_{2}$ are geometrically genus equivalent, it follows from Section 3.1, that $L$ is the maximal totally real field of $k_{2}$ and that $\left[k_{2}: L\right]=2$ also holds.

Any non-elementary Fuchsian subgroup of $\Gamma_{j}$ defines an associated quaternion algebra $B$ over $L$ that is an $L$-subalgebra of $A_{j}$. Conversely, any quaternion algebra $B / L$ that is unramified at the identity, ramified at all other real places of $L$, and for which $B \otimes_{L} k_{j} \cong A_{j}$, produces a commensurability class of arithmetic Fuchsian subgroups of $\Gamma_{j}$ (note that different embeddings of $B$ into $A_{j}$ can provide non-conjugate subgroups of $\Gamma_{j}$ ). Since $M_{1}$ and $M_{2}$ are geometrically genus equivalent, it follows that if $B$ is a quaternion algebra defined over $L$ that is unramified at the identity and ramified at all other real places of $L$, then:

$$
B \otimes_{L} k_{1} \cong A_{1} \text { if and only if } B \otimes_{L} k_{2} \cong A_{2} .
$$

In particular, it is easy to see that if $k_{1} \cong k_{2}$, then $A_{1} \cong A_{2}$ (after perhaps applying complex conjugation). Hence we are reduced to showing $k_{1} \cong k_{2}$.

To that end, let $R_{1}$ and $R_{2}$ be the set of finite places of $k_{1}$ and $k_{2}$ that ramify $A_{1}$ and $A_{2}, R_{j}^{\prime}$ denote those $L$-places lying below those places in $R_{j}$ for $j=1,2$, and let $V$ be the set of all finite places of $L$. Let $\omega \in V \backslash\left(R_{1}^{\prime} \cup R_{2}^{\prime}\right)$ be a place that is ramified or inert in $k_{1} / L$. Theorem 3.1 implies that we can construct a quaternion algebra $B$ over $L$ that is ramified at $R_{1}^{\prime} \cup \omega$ (and possibly other primes) and for which $B \otimes_{L} k_{1} \cong A_{1}$. Hence by (3.1), $B$ embeds in $A_{2}$. We claim that $\omega$ is ramified or inert in $k_{2}$. Indeed, if $\omega=\nu \nu^{\prime}$ is split in $k_{2}$, then $\left(k_{2}\right)_{\nu} \cong L_{\omega}$ and it follows that $A_{2} \otimes_{k_{2}}\left(k_{2}\right)_{\nu} \cong\left(B \otimes_{L} L_{\omega}\right)$ is a division algebra (and similarly for $\nu^{\prime}$ ). Hence $\nu, \nu^{\prime} \in R_{2}$, implying that $\omega \in R_{2}^{\prime}$, and this is a contradiction. Repeating this argument with the roles of $k_{1}$ and $k_{2}$ interchanged we deduce that apart from a finite number of $L$-primes, an $L$-prime is inert or ramified in $k_{1}$ if and only if it is inert of ramified in $k_{2}$. It follows then that apart from a finite number of $L$-primes, the splitting types in $k_{1}$ and $k_{2}$ are identical. Proposition 2.2 now implies $k_{1}=k_{2}$.

Note that the proof of Theorem 1.1 requires only the knowledge that if a commensurability class for a surface has a representative in $M_{1}$, then it also has a representative in $M_{2}$ (and conversely). We call a pair of manifolds $M_{1}, M_{2}$ with this property geometrically genus commensurable - compare again with the length spectrum setting $[17,1,16]$.

Theorem 3.2. Let $M_{1}=\mathbf{H}^{3} / \Gamma_{1}$ and $M_{2}=\mathbf{H}^{3} / \Gamma_{2}$ be arithmetic hyperbolic 3-manifolds. If $M_{1}$ and $M_{2}$ are geometrically genus commensurable then either they are commensurable or $G S\left(M_{1}\right)=\emptyset$. 


\section{Aligning surfaces: Theorem 1.3}

In this section, we prove Theorem 1.3. This contains most of the ideas that are needed in the proof of Theorem 1.2. The remaining complication in the proof of Theorem 1.2 will be controlling multiplicities.

4.1. Fuchsian pairs. The main idea in the proof of Theorem 1.3 is given by the following simple lemma.

Lemma 4.1. Let $M=\mathbf{H}^{3} / \Gamma$ be a finite volume hyperbolic 3-manifold.

(i) If $\Gamma_{1}$ and $\Gamma_{2}$ are (finite index) subgroups of $\Gamma$ such that

$$
\Gamma_{1} \cap F=\Gamma_{2} \cap F
$$

for every maximal Fuchsian subgroup $F$ of $\Gamma$, then the covers of $M$ corresponding to the subgroups $\Gamma_{1}$ and $\Gamma_{2}$ are geometrically genus equivalent.

(ii) If in addition $G$ is a finite group, $H$ and $K$ are subgroups of $G$, and $\phi: \Gamma \rightarrow G$ a surjective homomorphism such that

$$
H \cap \phi(F)=K \cap \phi(F)
$$

for every maximal Fuchsian subgroup $F$ of $\Gamma$, then the covers of $M$ corresponding to the subgroups $\phi^{-1}(H)$ and $\phi^{-1}(K)$ are geometrically genus equivalent.

Proof. (ii) follows immediately from (i). That (i) holds follows, since as noted earlier, the geometric genus set of $M$ is determined by the maximal Fuchsian subgroups of its associated Kleinian group.

We call a pair of finite groups $H, K<G$ a Fuchsian pair with respect to $\phi$ if $(4.2)$ holds.

4.2. Geometrical genus equivalence without volume control. We begin the proof of Theorem 1.3 by proving the following which is nothing more than Theorem 1.3 without volume considerations. The additional volume constraint will be addressed in Section 4.3.

Theorem 4.2. Let $M=\mathbf{H}^{3} / \Gamma$ be an arithmetic hyperbolic 3-manifold. Then there exists infinitely many pairs of non-isometric finite covers $\left(M_{j}, N_{j}\right)$ of $M$ such that $M_{j}, N_{j}$ are geometrically genus equivalent and non-isometric.

Proof. Without loss of generality we can pass to a subgroup of finite index so that the invariant trace-field $k$ of $\Gamma$ coincides with the trace-field. For simplicity, we will continue to denote this group by $\Gamma$. Recall that Theorem 3.1 shows that the invariant trace-field of any maximal Fuchsian subgroup $F$ of $\Gamma$ coincides with the maximal totally real subfield $L=k \cap \mathbf{R}$ and $[k: L]=2$.

By the Strong Approximation Theorem (see [15] or [20]) and Lemma 2.3, there exists a positive density set of places $\mathcal{P}$ of $k$ such that for each place $\nu \in \mathcal{P}$ and place $\omega$ of $L$ with $\nu \mid \omega, \phi_{\nu}(\Gamma)=\operatorname{PSL}\left(2, \mathbf{F}_{q_{\nu}}\right)$, the extension $\mathbf{F}_{q_{\nu}} / \mathbf{F}_{q_{\omega}}$ is proper (that is, it has degree 2), and the extensions $\mathbf{F}_{q_{\omega}} / \mathbf{F}_{p_{\nu}}$ (the latter being the prime field) is of some fixed degree $j$. For convenience we shall omit any dyadic places from $\mathcal{P}$. 
We will apply Lemma 4.1 in the following way. For each place $\nu \in \mathcal{P}$, we seek a non-trivial subgroup $C_{\nu}$ of $\operatorname{PSL}\left(2, \mathbf{F}_{q_{\nu}}\right)$ such that $C_{\nu} \cap \phi_{\nu}(F)$ is trivial for all maximal Fuchsian subgroups $F$ of $\Gamma$. Given this, the subgroup $C_{\nu}$ together with the trivial subgroup $\{1\}$ form a Fuchsian pair with respect to $\phi_{\nu}$, and so by Lemma 4.1, the covers $M_{C_{\nu}}$ and $M_{1, \nu}$ of $M$ corresponding to the subgroups $\phi_{\nu}^{-1}\left(C_{\nu}\right)$ and $\phi_{\nu}^{-1}(\{1\})$ are geometrically genus equivalent. Furthermore, note that the manifolds $M_{C_{\nu}}$ and $M_{1}$ cannot be isometric since $M_{1, \nu}$ properly covers $M_{C_{\nu}}$.

The subgroup $C_{\nu}$ is provided by the following lemma, the proof of which is given below.

Lemma 4.3. There exists a prime $\ell_{\nu}$ that divides the order of $\operatorname{PSL}\left(2, \mathbf{F}_{q_{\nu}}\right)$ but not the orders of $\operatorname{PSL}\left(2, \mathbf{F}_{q_{\omega}}\right)$ or $\mathbf{B}\left(\mathbf{F}_{q_{\omega}}(\sqrt{b})\right)$.

Given the lemma, Cauchy's theorem provides an element $\lambda_{\nu}$ of order $\ell_{\nu}$ in $\operatorname{PSL}(2$, $\left.\mathbf{F}_{q_{\nu}}\right)$, and hence a cyclic subgroup $\left\langle\lambda_{\nu}\right\rangle=C_{\nu}$ of $\operatorname{PSL}\left(2, \mathbf{F}_{q_{\nu}}\right)$ of order $\ell_{\nu}$. That this satisfies the condition $C_{\nu} \cap \phi_{\nu}(F)=1$ for any maximal Fuchsian subgroup follows from Lemma 2.4 and elementary group theory.

Proof of Lemma 4.3. Recall that

$$
\begin{aligned}
& \left|\operatorname{PSL}\left(2, \mathbf{F}_{q_{\omega}}\right)\right|=\frac{q_{\omega}\left(q_{\omega}^{2}-1\right)}{2}=\frac{q_{\omega}\left(q_{\omega}+1\right)\left(q_{\omega}-1\right)}{2}, \\
& \left|\mathbf{B}\left(\mathbf{F}_{q_{\omega}}(\sqrt{b})\right)\right|=\frac{q_{\omega}^{2}\left(q_{\omega}^{2}-1\right)}{2}=\frac{q_{\omega}^{2}\left(q_{\omega}+1\right)\left(q_{\omega}-1\right)}{2}, \\
& \left|\operatorname{PSL}\left(2, \mathbf{F}_{q_{\nu}}\right)\right|=\frac{q_{\omega}^{2}\left(q_{\omega}^{2}+1\right)\left(q_{\omega}^{2}-1\right)}{2}=\frac{q_{\nu}\left(q_{\nu}+1\right)\left(q_{\nu}-1\right)}{2} .
\end{aligned}
$$

Since the numbers $q_{\nu}+1, q_{\omega}$ are relatively prime and the numbers $q_{\nu}-1, q_{\nu}+1$ share only 2 as a common prime divisor, Lemma 4.3 can be established by finding an odd prime divisor of $q_{\nu}+1$. However, this is elementary as $q_{\nu}+1=2 \alpha_{\nu}$ where $\alpha_{\nu} \geq 5$ is odd. Thus any prime divisor $\ell_{\nu}$ of $\alpha_{\nu}$ will suffice for the lemma.

\section{Remarks:}

(1) There is an obvious similarity between the statement of Lemma 4.1 and a basic lemma used in the methods of Sunada $[18,9]$. Thus our method can be viewed as a generalization of Sunada's method to the setting of geometric genus spectra. However, unlike the case of Sunada our method is not purely algebraic. The reason is this: in Sunada's method, $\pi_{1}\left(S^{1}\right)$ is cyclic, so under any homomorphism $\phi$ of $\Gamma$, the image of $\pi_{1}\left(S^{1}\right)$ is cyclic, and so $\phi$ plays a minimal role while the role of $G$ is paramount. In contrast, in applying Lemma 4.1, one must have knowledge of the image of Fuchsian subgroups of $\Gamma$ under $\phi$, images which could be extremely complicated.

(2) Although Lemma 4.1 is stated only for totally geodesic surfaces, one can formulate a statement for surface subgroups of Kleinian groups more generally. Indeed, recent work of Kahn and Markvoic [7] shows that closed hyperbolic 3-manifolds contain many $\pi_{1}$-injective closed orientable surfaces that are not totally geodesic. Thus, one could attempt to employ Lemma 4.1 in this context. However, there seems to be no way of controlling where the image of a general surface group maps under any homomorphism onto a finite group. Indeed, when the trace-field of the surface group coincides with that of the Kleinian group (which is the typical situation when the 
surface subgroup is not Fuchsian), the Strong Approximation Theorem ensures that the restriction of $\phi_{\nu}$ to the surface group is surjective for all but finitely many places.

The terminology geometric genus spectrum is meant to emphasize that the free homotopy classes of surfaces in the 3-manifold under consideration are of a geometric origin. In light of the previous paragraph, the genus spectrum of $M$ is the set of pair $\left((g, n), \ell_{g, n}(M)\right)$ of free homotopy classes of $\pi_{1}$-injective, properly immersed surfaces of $M$ that contain a representative of topological type $\Sigma_{g, n}$. By [19] Corollary 8.8.6, if $M$ is a closed hyperbolic 3-manifold then again $\ell_{g, n}(M)$ is finite. Indeed, Kahn and Markovic[8] prove that the number of such genus $g$ surfaces is asymptotically $g^{2 g}$. It is an interesting problem to see how much of the topology and geometry of $M$ is determined by this topological spectrum.

4.3. Ensuring volume growth: Proof of Theorem 1.3. We now show how to extend the method of proof of Theorem 4.2 to obtain Theorem 1.3.

Proof of Theorem 1.3. The proof of Lemma 4.3 shows that any odd prime divisor $\ell_{\nu}$ of $q_{\nu}+1$ produces a pair of geometrically genus equivalent covers with volume ratio $\ell_{\nu}$. Ranging over $\mathcal{P}$, if the set of $\ell_{\nu}$ is unbounded, then the result follows. Otherwise, there is a finite list of primes $\ell_{1}, \ldots, \ell_{r}$ such that (recall $q_{\nu}+1$ is never zero modulo 4 )

$$
q_{\nu}+1=2 \prod_{i=1}^{r} \ell_{i}^{\alpha_{i, \nu}} .
$$

Note that $q_{\nu}$ and the exponents $\alpha_{i, \nu}$ depend on the place $\nu \in \mathcal{P}$ but the primes $\ell_{1}, \ldots, \ell_{r}$, by assumption, do not. In particular, since the left-hand side is unbounded as we range over $\mathcal{P}$, one of the exponents $\alpha_{i_{0}, \nu}$ must be unbounded as we range over $\mathcal{P}$. According to Lemma 2.1, since $\ell_{i}^{\alpha_{i_{0}, \nu}}$ divides $q_{\nu}+1$, there exists an element $\lambda_{\nu}$ of order $\ell_{i}^{\alpha_{i_{0}, \nu}}$ in $\operatorname{PSL}\left(2, \mathbf{F}_{q_{\nu}}\right)$. Arguing as before, we see that the subgroup $C_{\nu}$ generated by $\lambda_{\nu}$ and the trivial subgroup form a Fuchsian pair for $\phi_{\nu}$. The resulting geometrically genus equivalent covers of $M$ have a volume ratio of $\ell_{i}^{\alpha_{i_{0}, \nu}}$, which by choice of $\alpha_{i_{0}, \nu}$, is unbounded as we range over $\mathcal{P}$.

Remark: The manifolds $M_{C_{\nu}}$ and $M_{1, \nu}$ in the proof of Theorem 4.2 are not geometrically isogenus when $\operatorname{GS}(M) \neq \emptyset$. To see this, note that the maximal orientable totally geodesic surfaces up to free homotopy in a hyperbolic 3-manifold $M=\mathbf{H}^{3} / \Gamma$ are in bijection with the $\Gamma$-conjugacy classes of maximal Fuchsian subgroups of $\Gamma$. Thus given a finite cover $N \rightarrow M$, the maximal orientable totally geodesic surfaces up to free homotopy in $N$ are parameterized by the $\pi_{1}(N)$-conjugacy classes of the Fuchsian subgroups $\pi_{1}(N) \cap F$, where $F$ is a maximal Fuchsian subgroup of $\Gamma$.

Applying this discussion to the manifolds $M_{C_{\nu}}$ and $M_{1, \nu}$, we know that $\pi_{1}\left(M_{C_{\nu}}\right)$ and $\pi_{1}\left(M_{1, \nu}\right)$ satisfy (4.1). Since $M_{1, \nu} \rightarrow M_{C_{\nu}}$ is a cyclic cover of degree $\ell_{\nu}$ and (4.1) holds, each $\pi_{1}\left(M_{C_{\nu}}\right)$-conjugacy class $\pi_{1}\left(M_{C_{\nu}}\right) \cap F$ produces $\ell_{\nu}$ distinct $\pi_{1}\left(M_{1, \nu}\right)$ conjugacy classes in $\pi_{1}\left(M_{1, \nu}\right)$. This yields the relationship:

$$
\mathcal{G} S\left(M_{\nu, 1}\right)=\left\{\left(X, \ell_{X}\left(M_{\nu, 1}\right)\right\}=\left\{\left(X, \ell_{\nu} \cdot \ell_{X}\left(M_{\nu, \ell_{\nu}}\right)\right)\right\} .\right.
$$

In particular, none of the non-zero multiplicities are the same in the geometric genus spectra. However, the uniform nature of this failure will provide us a handle for matching up multiplicities for other pairs of covers. 


\section{Arranging multiplicities: Proof of Theorem 1.2}

In this section, we prove Theorem 1.2. As discussed above, the proof of Theorem 4.2 produces infinitely many pairs of finite covers $M_{C_{\nu}}$ and $M_{1, \nu}$ that are geometrically genus equivalent with a very precise relationship between their geometric genus spectra given by (4.3). We now show how to exploit (4.3) to produce infinitely many pairs of geometrically isogenus manifolds by using product homomorphisms $\phi_{\nu_{1}} \times \phi_{\nu_{2}}$. More precisely, Theorem 1.2 follows from (using the notation from the proof of Theorem 4.2):

Proposition 5.1. There exists an infinite subset of places $\nu \in \mathcal{P}$ for which a fixed prime $\ell$ can be taken for $\ell_{\nu}$.

Deferring the proof of this we complete the proof of Theorem 1.2.

Proof of Theorem 1.2. Let $\mathcal{P}^{\prime} \subset \mathcal{P}$ be the subset produced by Proposition 5.1 for the prime $\ell$. For any pair of places $\nu_{1}, \nu_{2} \in \mathcal{P}^{\prime}$, we have a pair of surjective homomorphisms $\phi_{\nu_{j}}: \Gamma \rightarrow \operatorname{PSL}\left(2, \mathbf{F}_{q_{\nu_{j}}}\right)$ such that $\ell$ divides the order of $\operatorname{PSL}\left(2, \mathbf{F}_{q_{\nu_{j}}}\right)$ but not the orders of PSL(2, $\left.\mathbf{F}_{q_{\omega_{j}}}\right)$ or $\mathbf{B}\left(\mathbf{F}_{q_{\omega_{j}}}(\sqrt{b})\right)$. Let $C_{\nu_{j}}$ be a cyclic subgroup of $\operatorname{PSL}\left(2, \mathbf{F}_{q_{\nu_{j}}}\right)$ of order $\ell$. Setting

$$
\phi_{1,2}: \Gamma \rightarrow \operatorname{PSL}\left(2, \mathbf{F}_{q_{\nu_{1}}}\right) \times \operatorname{PSL}\left(2, \mathbf{F}_{q_{\nu_{2}}}\right)
$$

to be the product homomorphism $\phi_{\nu_{1}} \times \phi_{\nu_{2}}$ (which is also surjective), we define subgroups $H_{1}=C_{\nu_{1}} \times\{1\}$ and $H_{2}=\{1\} \times C_{\nu_{2}}$. By construction of $\ell$, it follows that $H_{1}, H_{2}$ satisfy (4.2), and thus form a Fuchsian pair for $\phi_{1,2}$. Hence, by Lemma 4.1(ii), the covers $M_{\nu_{1}, \nu_{2}, H_{1}}, M_{\nu_{1}, \nu_{2}, H_{2}}, M_{\nu_{1}, \nu_{2},\{1\}}$ associated to the subgroups $\phi_{1,2}^{-1}\left(H_{1}\right), \phi_{1,2}^{-1}\left(H_{2}\right), \phi_{1,2}^{-1}(\{1\})$ are pairwise geometrically genus equivalent. Indeed, by $(4.3)$

$$
\mathcal{G} S\left(M_{\nu_{1}, \nu_{2},\{1\}}\right)=\left\{\left(X, \ell \cdot \ell_{X}\left(M_{\nu_{1}, \nu_{2}, H_{j}}\right)\right)\right\}
$$

for $j=1,2$. In particular,

$$
\ell \cdot \ell_{X}\left(M_{\nu_{1}, \nu_{2}, H_{1}}\right)=\ell \cdot \ell_{X}\left(M_{\nu_{1}, \nu_{2}, H_{2}}\right)
$$

and thus

$$
\ell_{X}\left(M_{\nu_{1}, \nu_{2}, H_{1}}\right)=\ell_{X}\left(M_{\nu_{1}, \nu_{2}, H_{2}}\right)
$$

It remains to prove that $M_{\nu_{1}, \nu_{2}, H_{1}}, M_{\nu_{1}, \nu_{2}, H_{2}}$ are not isometric. If this were not the case, then $\pi_{1}\left(M_{\nu_{1}, \nu_{2}, H_{1}}\right)$ and $\pi_{1}\left(M_{\nu_{1}, \nu_{2}, H_{2}}\right)$ would be conjugate in $\operatorname{Isom}\left(\mathbf{H}^{3}\right)$. We claim this is impossible. To that end, set $\lambda_{\nu_{1}} \in C_{\nu_{1}}$ to be a generator and $\gamma_{\nu_{1}} \in \pi_{1}\left(M_{\nu_{1}, \nu_{2}, H_{1}}\right)$ such that $\phi_{1}\left(\gamma_{\nu_{1}}\right)=\lambda_{\nu_{1}}$. Recall that the homomorphisms $\phi_{\nu}$ arose from the reduction of $R_{\nu}$ be the unique maximal ideal $\pi R_{\nu}$ and so induce homomorphisms $\phi_{\nu}: R_{\nu}[t] \rightarrow \mathbf{F}_{q_{\nu}}[t]$. It is a simple matter that for $\eta \in \operatorname{PSL}\left(2, R_{\nu}\right)$, we have the relationship $c_{\phi_{\nu}(\eta)}(t)=\phi_{\nu}\left(c_{\eta}(t)\right)$, where $c_{\theta}(t) \in R_{\nu}[t]$ denotes the characteristic polynomial of $\theta$.

By definition, every element $\gamma$ of $\pi_{1}\left(M_{\nu_{1}, \nu_{2}, H_{2}}\right)$ maps trivially under $\phi_{\nu_{1}}$ and thus $\phi_{\nu_{1}}\left(c_{\gamma}(t)\right)=(t-1)^{2}$. As conjugation by any $\tau$ in $\operatorname{Isom}\left(\mathbf{H}^{3}\right)$ preserves determinant and at worst changes trace by complex conjugation, any $\operatorname{Isom}\left(\mathbf{H}^{3}\right)$-conjugate of $\gamma$ also has this property. In particular, if $\gamma_{\nu_{1}}$ is $\operatorname{Isom}\left(\mathbf{H}^{3}\right)$-conjugate into $\pi_{1}\left(M_{\nu_{1}, \nu_{2}, H_{2}}\right)$, it would have to be that $c_{\lambda_{\nu_{1}}}(t)=(t-1)^{2}$. However, this is impossible since $\lambda_{\nu_{1}}$ is a semisimple element of odd prime order $\ell$. 
To produce infinitely many pairs, we can simply vary $\nu_{1}, \nu_{2}$ over $\mathcal{P}^{\prime} \times \mathcal{P}^{\prime} \backslash \Delta$ where $\Delta$ denotes the diagonal.

Proof of Proposition 5.1. In the proof of the proposition, we will keep with the notation used in the proof of Theorem 4.2. For the reader's convenience, we will briefly recall some of the notation here. Recall that we restricted ourselves to a positive density subset $\mathcal{P}$ of places of $k$ in order to control $\left[\mathbf{F}_{q_{\nu}}: \mathbf{F}_{q_{\omega}}\right]$ and $\left[\mathbf{F}_{q_{\omega}}: \mathbf{F}_{p_{\nu}}\right]$ and will continue to do so here. In addition, $q_{\nu}, q_{\omega}, p_{\nu}$ will denote the orders of the field $\mathbf{F}_{q_{\nu}}, \mathbf{F}_{q_{\omega}}$, and $\mathbf{F}_{p_{\nu}}$, and $\mathrm{P}$ will denote the set of all integral primes.

For each $\nu \in \mathcal{P}$, we saw from the proof of Theorem 4.2 that $\ell_{\nu}$ can be any odd prime divisor of $q_{\nu}+1$. What is required here is to find an odd prime $\ell$ and an infinite subset $\mathcal{P}^{\prime} \subset \mathcal{P}$ such that $\ell \mid q_{\nu}+1$ for all $\nu \in \mathcal{P}^{\prime}$. In fact, we will find an odd prime $\ell$ and a positive density (and hence infinite) subset $\mathcal{P}^{\prime}$ for which this holds. The remainder of this proof is devoted to this task.

Recall that $q_{\nu}=q_{\omega}^{2}=p_{\nu}^{2 j}$ where $j$ is fixed, and so $q_{\nu}+1=p_{\nu}^{2 j}+1$. It is elementary that for any (odd) prime $\ell$, if $\ell$ divides $p_{\nu}^{2 j}+1$, then $p_{\nu}^{2 j}+1 \equiv 0 \bmod \ell$. Equivalently, setting $F_{j}(t)=t^{2 j}+1$, the previous statement is simply that $\overline{p_{\nu}}$ is a zero of $F_{j}(t)$ in $\mathbf{F}_{\ell}$ (where $\overline{p_{\nu}}$ is the modulo $\ell$ residue class of $p_{\nu}$ in $\mathbf{F}_{\ell}$ ).

We now show how to use $F_{j}(t)$ and the Chebotarev density theorem to find the required $\ell$ and $\mathcal{P}^{\prime}$. To that end, let $\mathcal{L}_{j}$ be the set of odd primes $\ell$ such that $F_{j}(t)$ has a root in $\mathbf{F}_{\ell}$. For each $\ell \in \mathcal{L}_{j}$, define the set

$$
\mathrm{P}_{\ell}^{j}=\left\{m \in \mathbf{N}: F_{j}(\bar{m})=0 \quad \bmod \ell\right\} \cap \mathrm{P} .
$$

By the Chebotarev Density Theorem,

$$
\operatorname{Density}\left(\mathrm{P}_{\ell}^{j}\right) \geq \frac{1}{\ell-1}
$$

In addition, since for every prime $p$, there exists an odd prime divisor of $p^{2 j}+1$ (recall that $p^{2 j}+1$ is never zero modulo 4 ),

$$
\bigcup_{\ell \in \mathcal{L}_{j}} \mathrm{P}_{\ell}^{j}=\mathrm{P} .
$$

After ordering $\mathcal{L}_{j}=\left\{\ell_{1}<\ell_{2}<\ell_{3}<\cdots\right\}$, for each $i \geq 1$, we define the set

$$
A_{i}=\bigcup_{m=1}^{i} \mathrm{P}_{\ell_{m}}^{j} .
$$

The sequence of densities $\left\{\operatorname{Density}\left(A_{i}\right)\right\}_{i}$ for the sets $A_{i}$ is positive by $(5.1)$, strictly increasing (since $\mathrm{P}_{\ell_{i}}^{j} \cap \mathrm{P}_{\ell_{i^{\prime}}}^{j}$ has density strictly smaller than $\mathrm{P}_{\ell_{i}}^{j}$ and $\mathrm{P}_{\ell_{i^{\prime}}}^{j}$ for all distinct $\left.i, i^{\prime}\right)$, and has a least upper bound of 1 by (5.2). This in tandem with the fact that the set

$$
\mathrm{P}_{\mathcal{P}}=\left\{p_{\nu}: \nu \in \mathcal{P}\right\}
$$

has positive density, implies that there exists an $i_{0}$ such that $A_{i_{0}} \cap \mathrm{P}_{\mathcal{P}}$ has positive density. By the Pigeonhole Principle, there exists $1 \leq i_{1} \leq i_{0}$ such that

$$
\mathrm{P}^{\prime}=\mathrm{P}_{\ell_{i_{1}}}^{j} \cap \mathrm{P}_{\mathcal{P}}
$$


has positive density. In particular, for each $p \in \mathrm{P}^{\prime}$, the prime $\ell_{i_{1}}$ divides $p^{2 j}+1$. Thus, $\ell_{i_{1}}$ and the set

$$
\mathcal{P}^{\prime}=\left\{\nu \in \mathcal{P}: p_{\nu} \in \mathrm{P}^{\prime}\right\}
$$

is the required pair needed to finish the proof.

\section{Examples}

In this section, we discuss our constructions in the context of some examples.

6.1. Take $k=\mathbf{Q}(i)$ and $L=\mathbf{Q}$. Since any prime $p \equiv 3 \bmod 4$ is inert in $k$, we see that $\mathbf{F}_{\nu} \neq \mathbf{F}_{p}$ for the unique place $\nu$ dividing $p$. Consider the primes 3 and 7 . Note that $\left|\operatorname{PSL}\left(2, \mathbf{F}_{9}\right)\right|=360$ and $\left|\operatorname{PSL}\left(2, \mathbf{F}_{49}\right)\right|=58800$ are both divisible by 5 . On the other hand $\left|\operatorname{PSL}\left(2, \mathbf{F}_{3}\right)\right|=12,\left|\operatorname{PSL}\left(2, \mathbf{F}_{7}\right)\right|=168,\left|\mathbf{B}\left(\mathbf{F}_{9}\right)\right|=36$ and $\left|\mathbf{B}\left(\mathbf{F}_{49}\right)\right|=1176$, all fail to have 5 as a divisor.

With this, let $A / k$ be any quaternion algebra which satisfies the hypothesis of Theorem 3.1 (and so contains Fuchsian subgroups) and for which the places of $k$ above 7 are not contained in $\operatorname{Ram}_{f} A$. Note that by definition of $A$, the place above 3 is not contained in $\operatorname{Ram}_{f} A$. Let $\mathcal{O}$ be an (maximal) order of $A$ and $\Gamma$ the image in $\operatorname{PSL}(2, \mathbf{C})$ of $\mathcal{O}^{1}$. In the notation of the proof of Theorem 4.2, we can take $\nu$ to be the place above 3 , and $\ell_{\nu}=5$ (so that $C_{\nu}$ is a cyclic group of order 5 ). The proof of Theorem 4.2 now produces geometrically genus equivalent covers of degree 72 and 360 , respectively.

To produce isogenus covers, we can take the places over 3 and 7 , and use $\ell=5$. The proof of Theorem 1.2 now produces geometrically isogenus covers of degree 4, 233, 600 .

We remark that the reduction homomorphisms do indeed surject $\Gamma$ onto the stated finite groups (without recourse to Strong Approximation). The reason for this is that since $\mathcal{O}$ is maximal, it will be dense in its localizations. Since $\nu$ is unramified, these localizations of $\mathcal{O}$ are isomorphic to $M\left(2, \mathbf{Z}[i]_{\nu}\right)$, and thus $\Gamma$ is dense in the groups $\operatorname{PSL}\left(2, \mathbf{Z}[i]_{\nu}\right)$ for the stated places $\nu$.

Note that there are infinitely many distinct commensurability classes of such examples that satisfy this condition on $\operatorname{Ram}_{f} A$. Indeed we can arrange for $\Gamma$ to be torsion-free.

6.2. Using the results of $[5,12]$ one can in principle obtain information on the topological types and multiplicities arising in the case of the groups $\operatorname{PSL}\left(2, O_{d}\right)$. These groups contain elements of finite order, but the arguments still apply. We sketch some of this for the case Picard group $\operatorname{PSL}\left(2, O_{1}\right)$.

Maximal arithmetic Fuchsian groups are parameterized by a positive integer $D$, called the discriminant. In [12] it is shown that the number of $\operatorname{PSL}\left(2, O_{1}\right)$-conjugacy classes of maximal arithmetic Fuchsian subgroups of discriminant $D$ is 1,2 or 3 dependent on whether $D$ is congruent to 0 or $3 \bmod 4,2 \bmod 4$ or $1 \bmod 4$. Indeed, one can get more detailed information on these arithmetic Fuchsian groups.

For all discriminants $D$, one conjugacy class is represented by the arithmetic Fuchsian group obtained as follows. Let $B_{D}$ be the indefinite quaternion algebra over $\mathbf{Q}$ with Hilbert Symbol $\left(\frac{-1, D}{\mathbf{Q}}\right)$ and $\mathcal{O}_{D}=\mathbf{Z}[1, i, j, i j]$. Then prescribing a particular 
representation $\rho: B_{D} \rightarrow M(2, \mathbf{C})$, the image of $\mathcal{O}_{D}^{1}$ determines an arithmetic Fuchsian subgroup which we denote by $F_{D}$. When $D$ is congruent to 0 or 3 modulo 4 this is the only such group (up to $\operatorname{PSL}\left(2, O_{1}\right)$-conjugacy).

In the case when $D$ is congruent to 1 modulo 4 , the two further conjugacy classes are represented by group $G_{D, 1}$ and $G_{D, 2}$. As remarked in [12], the subgroups $G_{D, 1}$ and $G_{D, 2}$ are conjugate in $\operatorname{PGL}\left(2, O_{1}\right)$. It is shown in [12] that the group $G_{D, 1}$ is the image in $\operatorname{PSL}\left(2, O_{1}\right)$ of the unit group of an order $\mathcal{M}_{D}$ of $B_{D}$ and $\left[\mathcal{M}_{D}^{1}: \mathcal{O}_{D}^{1}\right]=3$.

Finally for the case when $D$ is congruent to 2 modulo 4 , there is a further group $H_{D}$ that is described as the image of the unit group of another order $\mathcal{N}_{D}$ of $B_{D}$ and in this case $\left[\mathcal{N}_{D}^{1}: \mathcal{O}_{D}^{1}\right]=2$ or 6 dependent on whether $D$ is congruent to 2 or 6 modulo 8.

From this the co-volumes of these maximal arithmetic Fuchsian groups can be computed using a result of Humbert (see [12] Section 6). In addition, whether the group is non-cocompact is also decidable from $D$ (see Lemma 8 of [12]) and the numbers of conjugacy classes of elements of finite order (and the orders) are also computable (see [12] Section 7).

\section{The non-arithmetic case}

In this section, we outline how Theorems 1.2 and 1.3 can be extended to any finite volume hyperbolic 3-manifold.

Let $M=\mathbf{H}^{3} / \Gamma$ be a complete, finite volume hyperbolic 3 -manifold. Set $k$ to be the invariant trace field of $M$ and $F$ to be the maximal real subfield. For each place $\nu$ of $k$ and $\omega$ of $F$ with $\nu \mid \omega$, let $\mathbf{F}_{q_{\nu}}, \mathbf{F}_{q_{\omega}}$ be the associated residue fields and $t_{\nu}=\left[\mathbf{F}_{q_{\nu}}: \mathbf{F}_{q_{\omega}}\right]$. According to the Strong Approximation Theorem and Lemma 2.3, we can arrange it so that $t=t_{\nu}>1$ is constant, $\left[\mathbf{F}_{q_{\omega}}: \mathbf{F}_{p_{\nu}}\right]=j$ is constant, and $\phi_{\nu}(\Gamma)=\operatorname{PSL}\left(2, \mathbf{F}_{q_{\nu}}\right)$ for a positive density subset $\mathcal{P}$ of places $\nu$ of $k$.

The proof of Theorem 4.2 for general $M$ is done with precisely the same method. That Lemma 4.3 holds in this setting follows immediately from Zsigmondy's theorem [21]. However, to prove the analogue of Proposition 5.1 in this setting requires more than what Zsigmondy's theorem obviously gives, and with that in mind we therefore give more discussion. First, note that one can simplify things somewhat. For each $\nu \in \mathcal{P}$, take a subfield $\mathbf{F}_{q_{\omega}}<\mathbf{F}_{\nu}<\mathbf{F}_{q_{\nu}}$ such that the degree $\left[\mathbf{F}_{\nu}: \mathbf{F}_{q_{\omega}}\right]=p^{\prime}$ is a fixed prime. Notice that it certainly suffices to find a prime divisor $\ell_{\nu}$ of $\left|\operatorname{PSL}\left(2, \mathbf{F}_{\nu}\right)\right|$ that does not divide $\left|\operatorname{PSL}\left(2, \mathbf{F}_{q_{\omega}}\right)\right|$ or $\left|\mathbf{B}\left(\mathbf{F}_{q_{\omega}}(\sqrt{b})\right)\right|$ as this would also divide the order of the larger group $\operatorname{PSL}\left(2, \mathbf{F}_{q_{\nu}}\right)$. For $p^{\prime}=2$, this is nothing more than Lemma 4.3. However, when $p^{\prime}$ is odd, we need to amend the proof of Proposition 5.1 as follows. In this case, we can write

$$
\left|\operatorname{PSL}\left(2, \mathbf{F}_{\nu}\right)\right|=\frac{q_{\omega}^{p^{\prime}}\left(q_{\omega}+1\right)\left(q_{\omega}-1\right) r_{-, \nu} r_{+, \nu}}{2},
$$

where the factors $r_{ \pm, \nu}$ are given by:

$$
r_{+, \nu}=\frac{q_{\omega}^{p^{\prime}}+1}{q_{\omega}+1}, \quad r_{-, \nu}=\frac{q_{\omega}^{p^{\prime}}-1}{q_{\omega}-1},
$$

and have the following divisibility properties:

$$
\left(q_{\omega} \pm 1, r_{\mp, \nu}\right)=1, \quad\left(r_{ \pm, \nu}, q_{\omega}\right)=1, \quad\left(r_{+, \nu}, r_{-, \nu}\right)=1, \quad\left(r_{ \pm, \nu}, q_{\omega} \pm 1\right) \mid p^{\prime} .
$$


Assuming the validity of (7.2), the existence of $\ell_{\nu}$ is easy. As either $r_{+, \nu}$ or $r_{-, \nu}$ must be relatively prime to $p^{\prime}$ and both have at least one odd prime divisor, there exists an odd prime divisor of either $r_{+, \nu}$ or $r_{-, \nu}$ that does not divide $\left|\operatorname{PSL}\left(2, q_{\omega}\right)\right|$ and $\left|\mathbf{B}\left(\mathbf{F}_{q_{\omega}}(\sqrt{b})\right)\right|$.

The proof of (7.2) is straightforward. In fact, the first three assertions are trivial and only the proof of the last assertion requires comment. To begin, an elementary calculation shows that

$$
r_{-, \nu}=\sum_{j=0}^{p^{\prime}-1} q_{\omega}^{j}, \quad r_{+, \nu}=\sum_{j=1}^{p^{\prime}}(-1)^{p^{\prime}-j} q_{\omega}^{j-1} .
$$

Using this, we will work out the case of $\left(r_{-, \nu}, q_{\omega}-1\right)$. Let $\ell$ be a common divisor of $r_{-, \nu}$ and $q_{\omega}-1$. Then $\ell$ must divide

$$
\left(r_{-, \nu}+\left(q_{\omega}-1\right)\right)=\left(\sum_{j=0}^{p^{\prime}-1} q_{\omega}^{j}\right)+q_{\omega}-1=2 q_{\omega}+\sum_{j=2}^{p^{\prime}-1} q_{\omega}^{j} .
$$

From this, we see that that $\ell$ must divide

$$
\left(\left(2 q_{\omega}+\sum_{j=2}^{p^{\prime}-1} q_{\omega}^{j}\right)+2 q_{\omega}\left(q_{\omega}-1\right)\right)=3 q_{\omega}^{2}+\sum_{j=3}^{p^{\prime}-1} q_{\omega}^{j} .
$$

Using this procedure, we can now inductively deduce:

$$
\ell \mid\left(m q_{\omega}^{m-1}+\sum_{j=m}^{p^{\prime}-1} q_{\omega}^{j}\right)
$$

for any integer $2 \leq m \leq p^{\prime}-1$. Taking $m=p^{\prime}-1$ in (7.3) yields that $\ell$ must divide

$$
\left(1-p^{\prime}\right) q_{\omega}^{p^{\prime}-2}+q_{\omega}^{p^{\prime}-1}=q_{\omega}^{p^{\prime}-2}\left(q_{\omega}+p^{\prime}-1\right)
$$

and thus $\ell \mid\left(q_{\omega}+p^{\prime}-1\right)$. This in tandem with the fact that $\ell \mid\left(q_{\omega}-1\right)$ implies that $\ell \mid p^{\prime}$ as desired. For the case of $\ell \mid\left(r_{+, \nu}, q_{\omega}+1\right)$, the corresponding inductive statement becomes:

$$
\ell \mid\left((-1)^{p^{\prime}-m} m q_{\omega}^{m-1}+\sum_{j=m+1}^{p^{\prime}}(-1)^{p^{\prime}-j} q_{\omega}^{j-1}\right)
$$

for all $2 \leq m \leq p^{\prime}-1$. Taking $m=p^{\prime}-1$ in (7.4) implies that $\ell$ divides

$$
\left(-p^{\prime}+1\right) q_{\omega}^{p^{\prime}-2}+q_{\omega}^{p^{\prime}-1}=q_{\omega}\left(1-p^{\prime}+q_{\omega}\right) .
$$

As before, this and $\ell \mid q_{\omega}+1$ implies that $\ell \mid p^{\prime}$.

To prove the extension of Theorem 1.3 in this case, what is important here is that the prime $\ell_{\nu}$ obtained from the extension of Lemma 4.3 occurs as a prime divisor of one of $r_{+, \nu}, r_{-, \nu}$. Taking a positive density subset of places $\nu$ where the $\ell_{\nu}$ arises as a factor of a fixed $r_{\star, \nu}$, the argument is identical.

For the extension of Theorem 1.2, we replace the polynomial $x^{2 j}+1$ by one associated to either $r_{+, \nu}$ or $r_{-, \nu}$ obtained from (7.1) (at least one occurs with positive density). With this, the argument is identical to that given in the proof of Proposition 5.1. 


\section{Final remarks and questions}

In this final section, we collect some questions that naturally arise from this work.

8.1. Geometric genus spectrum invariants. All of the examples of geometrically isogenus manifolds produced in Theorem 1.2 have equal volume. As this is a well known spectral invariant, this prompts the question:

Question. Do geometrically isogenus (arithmetic) hyperbolic 3-manifolds with nontrivial geometric genus spectra always have equal volume?

8.2. A criteria for arithmeticity. As Theorem 1.3 shows, any pair of arithmetic hyperbolic 3-manifolds with identical, non-trivial geometric genus sets are commensurable. As already discussed, we do not know of a non-arithmetic example for which geometric genus set is infinite.

Question. If the geometric genus set of a complete, finite volume hyperbolic 3manifold $M$ is infinite, is $M$ arithmetic?

8.3. Commensurability in general. As we expect non-arithmetic complete, finite volume hyperbolic 3-manifolds to typically have finite geometric genus set, a generalization of Theorem 1.1 to non-arithmetic manifolds seems unlikely. In particular, we expect an affirmative answer to the following question.

Question. Do there exist incommensurable non-arithmetic 3-manifolds with equal, non-trivial geometric genus sets? What about equal non-trivial geometric genus spectra?

A class of examples which are good candidates for an affirmative answer to these questions are certain non-arithmetic hyperbolic twist knot complements. Any hyperbolic twist knot complement contains an immersed twice punctured disk which is always totally geodesic with a unique hyperbolic structure (arising from the level 2 principal congruence subgroup of $\operatorname{PSL}(2, \mathbf{Z})$ ). Now using [4] we can arrange for infinitely many of these twist knot complements to have invariant trace-fields of odd prime degree. It now follows from Theorem 5.3.8 of [11] that these knot complements contain no closed totally geodesic surfaces, and furthermore the proof shows that any totally geodesic surface in the knot complement covers $\mathbf{H}^{2} / \operatorname{PSL}(2, \mathbf{Z})$.

At present we cannot rule out the existence of other surfaces but we believe that the totally geodesic twice punctured disk (and its covers) are the only totally geodesic surfaces in these knot complements.

\section{Acknowledgments}

Both authors wish to thank R.I.M.S. Kyoto for their hospitality in December 2006 when this work began. Additionally, the first author wishes to thank the California Institute of Technology as some of this work was done during an extended visit to that institution, and the second author wishes to thank the Institute for Advanced Study where this work was completed. The first author was partially supported by an NSF postdoc and the second author was partially supported by an NSF grant. 


\section{References}

[1] T. Chinburg, E. Hamilton, D.D. Long and A.W. Reid, Geodesics and commensurability classes of arithmetic hyperbolic 3-manifolds, Duke Math. J. 145 (2008), 25-44.

[2] J.J. Duistermaat and V.W. Guillemin, The spectrum of positive elliptic operators and periodic geodesics, Invent. Math. 29 (1975), 39-79.

[3] R. Gangolli, The length spectra of some compact manifolds, J. Differ. Geom. 12 (1977), 403424.

[4] J. Hoste and P.D. Shanahan, Trace fields of twist knots, J. Knot Theory Ramifications 10 (2001), 625-639.

[5] D.G. James and C. Maclachlan, Fuchsian subgroups of Bianchi groups, Trans. Amer. Math. Soc. 348 (1996), 1989-2002.

[6] G.J. Janusz, Algebraic number fields, Second Edn., Graduate Studies in Math. 7, A.M.S (1996).

[7] J. Kahn and V. Markovic, Immersing almost geodesic surfaces in a closed hyperbolic three manifold, Ann. Math. (2) 175 (2012), 1127-1190.

[8] J. Kahn and V. Markovic, Counting essential surfaces in a closed hyperbolic three-manifold, Geom. Topol. 16 (2012), 601-624.

[9] C.J. Leininger, D.B. McReynolds, W.D. Neumann, and A.W. Reid, Length and eigenvalue equivalence, Int. Math. Research Notices 24 (2007), 24 pp.

[10] A. Lubotzky, B. Samuels and U. Vishne, Division algebras and non-commensurable isospectral manifolds, Duke Math. J. 135 (2006), 361-379.

[11] C. Maclachlan and A.W. Reid, The arithmetic of hyperbolic 3-manifolds, Graduate Texts in Mathematics 219, Springer-Verlag (2003).

[12] C. Maclachlan and A.W. Reid, Parametrizing Fuchsian subgroups of Bianchi groups, Can. J. Math. 43 (1991), 158-181.

[13] D.B. McReynolds, Geometric spectra and commensurability, to appear in Cand. Jour Math., http://front.math.ucdavis.edu/1401.1516

[14] J.S. Meyer, A division algebra with infinite genus, http://front.math.ucdavis.edu/1301. 5632

[15] M.V. Nori, On subgroups of $\mathrm{GL}_{n}\left(\mathbf{F}_{p}\right)$, Invent. Math. 88 (1987), 257-275.

[16] G. Prasad and A. Rapinchuk, Weakly commensurable arithmetic groups and isospectral locally symmetric spaces, Publ. Math. Inst. Hautes Études Sci. 109 (2009), 113-184.

[17] A.W. Reid, Isospectrality and commensurability of arithmetic hyperbolic 2- and 3-manifolds, Duke Math. J. 65 (1992), 215-228.

[18] T. Sunada, Riemannian coverings and isospectral manifolds, Ann. Math. 121 (1985), 169186.

[19] W.P. Thurston, The Geometry and Topology of 3-manifolds, Princeton University Mimeographed Notes (1979).

[20] B. Weisfeiler, Strong approximation for Zariski-dense subgroups of semisimple algebraic groups, Ann. Math. 120 (1984), 271-315.

[21] K. Zsigmondy, Zur theorie der Potenzreste, Monatsh. Für Math. Phys. 3 (1892), 265-284.

Department of Mathematics, Purdue University, West Lafayette, IN 47906, USA

E-mail address: dmcreyno@math.purdue.edu

Department of Mathematics, University of Texas, Austin, TX 78712, USA

E-mail address: areid@math.utexas.edu 
